\title{
Une moindre exposition des sous-mariniers au rayonnement naturel
}

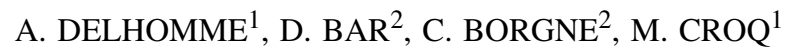

(Manuscrit reçu le 18 janvier 2010, accepté le 17 août 2010)

RÉSUMÉ Les spectres d'anthroporadiamétrie des sous-mariniers français embarqués sur sous-marins nucléaires lanceurs d'engins (SNLE) mettent en évidence une activité corporelle globale plus faible au retour de patrouille que lorsqu'ils sont à terre. Cette différence s'explique par l'absence de radon à bord des sous-marins après plusieurs jours de patrouille. Cette absence de radon conduit à une diminution de l'exposition des sous-mariniers à la principale source de rayonnement naturel. Par ailleurs, les conditions de plongée conduisent à une très forte atténuation des rayonnements tellurique et cosmique. En somme, les sous-mariniers embarqués sur SNLE présentent une moindre exposition aux principales sources d'exposition naturelle. Cette moindre exposition est globalement non compensée par un accroissement de l'exposition artificielle.

ABSTRACT A lower exposure to natural background radiation for submariners.

French submariners' whole-body counting spectra conducted in the first days following their return to naval base after a patrol on a SSBN clearly display a lower gross activity than when they are living at home. The explanation is quite simple: no source of radon exists aboard. Therefore, the naturally occurring contamination is nearly null. Furthermore, water strongly attenuates cosmic and terrestrial radiation. This leads to an underexposure of the French submariners to natural background radiation. This lack is only partially balanced by the artificial sources aboard.

Keywords: Submarine / radon / whole-body counting / natural radioactivity

\section{Introduction}

Les six équipages des sous-marins nucléaires lanceurs d'engins (SNLE) français bénéficient d'une surveillance de leur exposition aux rayonnements ionisants (Laroche, 2001 ; Delhomme, 2001). À la surveillance du risque d'exposition externe est associée une surveillance de l'exposition interne. Il est indispensable d'éliminer au plus tôt une éventuelle contamination par des radionucléides émetteurs gamma qui serait survenue durant la patrouille et n'aurait pas été révélée par les chaînes de détection. Pour cela un échantillon, composé de vingt personnes et représentatif de l'équipage, bénéficie d'un comptage anthroporadiamétrique

1 Marine nationale, service médical de l'escadrille des SNLE, 29240 Brest armées, France.

2 Marine nationale, laboratoire d'anthroporadiamétrie de la base de l'île longue, 29240 Brest armées, France. 
dans les quarante-huit heures qui suivent le retour. En situation normale, le reste de l'équipage effectue ce comptage dans les semaines qui suivent.

Une comparaison sommaire des spectres de l'échantillon avec ceux réalisés à distance du retour semblait montrer, et ce pour chaque équipage, une activité totale corporelle moindre pour les comptages effectués immédiatement après le retour.

La seule hypothèse envisageable est celle d'une moindre contamination des équipages par les radionucléides naturels en raison de l'absence de source de radon à bord des sous-marins. Pour tester et valider cette hypothèse une anthroporadiamétrie comparative, sur un échantillon de trente personnes rentrant de patrouille, a été réalisée.

Population : la série est composée de 31 membres d'un même équipage représentatifs des divers emplois rencontrés à bord d'un SNLE.

Perdus de vue : En raison de mutations professionnelles, deux individus de la série n'ont pas pu effectuer l'anthroporadiamétrie comparative.

\section{Matériel et méthode}

Tous les comptages ont été réalisés sur l'installation d'anthroporadiomètre de la base opérationnelle de l'île longue. Cette installation est de type Antariès, elle comprend deux détecteurs NaI 20 pouces.

L'examen est réalisé sur du personnel habillé.

Chaque individu a bénéficié d'un comptage initial sur 100 secondes dans les trente-six heures suivant le retour de mer, puis d'un comptage comparatif sur 100 secondes réalisé au moins trois semaines après.

Les spectres sommes nets ont été analysés grâce au logiciel InterWinner 6.0. En raison de l'absence de pic spécifique, l'analyse a consisté à réaliser l'intégrale du spectre dans la gamme d'énergie allant de $100 \mathrm{keV}$ à $2000 \mathrm{keV}$. Le choix de la valeur $100 \mathrm{keV}$ s'explique par un fort bruit électronique en-deçà de cette énergie associé à la mauvaise qualité de détection des NaI dans les basses énergies.

Soit « $\mathrm{d}$ » la différence entre le comptage comparatif « $\mathrm{c}$ » et le comptage initial « $\mathrm{i} »$. La comparaison des valeurs de « $\mathrm{d}$ » est réalisée au moyen d'un test unilatéral de Student. L'hypothèse testée est «la moyenne de d est nulle». L'hypothèse alternative est « la moyenne de d est strictement positive ».

La normalité de la distribution de « $\mathrm{d} »$ est vérifiée par un test de $\chi^{2}$. 
TABLEAU I

Résultats des comptages en nombre de coups.

Counting results.

\begin{tabular}{cccc}
\hline & \multicolumn{3}{c}{ Comptage } \\
Sujet & i & c & d \\
\hline $\mathbf{1}$ & 7638 & 8146 & 508 \\
$\mathbf{2}$ & 7503 & 7460 & -43 \\
$\mathbf{3}$ & 6540 & 6963 & 423 \\
$\mathbf{4}$ & 7283 & 7188 & -95 \\
$\mathbf{5}$ & 6028 & 6534 & 506 \\
$\mathbf{6}$ & 6922 & 6766 & -156 \\
$\mathbf{7}$ & 5984 & 6002 & 18 \\
$\mathbf{8}$ & 5678 & 5647 & -31 \\
\hline $\mathbf{9}$ & 6518 & 7631 & 1113 \\
$\mathbf{1 0}$ & 6185 & 6338 & 153 \\
\hline $\mathbf{1 1}$ & 6341 & 6589 & 248 \\
$\mathbf{1 2}$ & 7169 & 7262 & 93 \\
\hline $\mathbf{1 3}$ & 5544 & 6176 & 632 \\
\hline $\mathbf{1 4}$ & 5340 & 5441 & 101 \\
\hline $\mathbf{1 5}$ & 6576 & 9690 & 3114 \\
\hline $\mathbf{1 6}$ & 6406 & 6153 & -253 \\
\hline
\end{tabular}

\begin{tabular}{cccc}
\hline & \multicolumn{3}{c}{ Comptage } \\
Sujet & i & c & d \\
\hline $\mathbf{1 7}$ & 5422 & 5719 & 297 \\
$\mathbf{1 8}$ & 6803 & 8228 & 1425 \\
$\mathbf{1 9}$ & 7500 & 8060 & 560 \\
$\mathbf{2 0}$ & 6101 & 6626 & 525 \\
\hline $\mathbf{2 1}$ & 6646 & 6806 & 160 \\
\hline $\mathbf{2 2}$ & 6188 & 6470 & 282 \\
\hline $\mathbf{2 3}$ & 7376 & 6777 & -599 \\
\hline $\mathbf{2 4}$ & 6074 & 5981 & -93 \\
\hline $\mathbf{2 5}$ & 5248 & 6079 & 831 \\
\hline $\mathbf{2 6}$ & 7053 & 6902 & -151 \\
\hline $\mathbf{2 7}$ & 6616 & 5952 & -664 \\
\hline $\mathbf{2 8}$ & 5929 & 6137 & 208 \\
\hline $\mathbf{2 9}$ & 6323 & 6614 & 291 \\
& & & \\
\hline m & 6446 & 6770 & $\mathbf{3 2 4}$ \\
\hline s & 125 & 172 & $\mathbf{1 3 0}$ \\
\hline $\mathbf{m}:$ moyenne $;$ s : écart-type. & \\
\hline & & & \\
\hline
\end{tabular}

\section{Résultats}

Les résultats bruts sont donnés dans le tableau I.

Le test de $\chi^{2}$ sur la distribution de « $\mathrm{d}$ » donne $\chi_{0}^{2}=4,33$ pour une valeur seuil $\chi_{2 ; 0,05}^{2}=5,99$. On peut alors conclure qu'il n'y a pas de différence significative entre la distribution observée de « $\mathrm{d}$ » et sa distribution théorique. Les valeurs de « $\mathrm{d}$ étant normales, on peut appliquer le test de Student.

Ce dernier permet d'obtenir $\mathrm{t}_{0}=2,50$ qui est supérieur à $\mathrm{t}_{28 ; 0,05}=1,70$. La différence est significative au risque $\alpha=5 \%$. On peut ainsi rejeter l'hypothèse d'égalité des moyennes. L'activité totale corporelle est donc plus élevée après un séjour à terre qu'à l'issue d'une patrouille sur SNLE.

\section{Interprétation et discussion}

Pendant dix semaines, l'équipage d'un SNLE est soustrait à une partie des sources d'exposition naturelle. Pour ce qui est de l'exposition externe d'origine naturelle, la masse d'eau autour du sous-marin constitue un excellent écran contre les 
rayonnements telluriques et cosmiques. En ce qui concerne les sources d'exposition interne d'origine naturelle, la principale est le radon. Le sous-marin fabrique, filtre et élimine son air en continu. Ce système de renouvellement d'air conduit à une décroissance rapide du radon le plus problématique sur le plan sanitaire qu'est le ${ }^{222} \mathrm{Rn}$. Du fait de leurs demi-vies courtes, ses descendants sont en équilibre séculaire avec lui. Son élimination conduit ainsi à leur disparition (Charuau, 1996). Il semble alors cohérent que l'activité totale corporelle d'un sous-marinier au retour de patrouille soit moins forte qu'après un séjour de quelques semaines à terre.

Les anthroporadiamétries étant réalisées « habillé », une partie voire l'essentiel de la contamination par les descendants du radon visibles à l'examen, ${ }^{214} \mathrm{Bi}$ et ${ }^{214} \mathrm{~Pb}$, est déposée sur les vêtements. Le niveau de contamination des vêtements est un reflet indirect du niveau de contamination atmosphérique.

Pour le premier comptage de la série, certains sous-mariniers portaient des vêtements embarqués pour la patrouille et d'autres des vêtements provenant de leur domicile. Le premier comptage individuel est donc probablement surestimé pour certains. Toutefois cela n'est pas gênant pour l'interprétation puisque ce biais de mesure ne conduit pas à un test de Student non significatif. En revanche, cela explique la dispersion importante des valeurs de « $\mathrm{d} »$, y compris les valeurs de « $\mathrm{d} »$ négatives

En fonction des régions, le radon est à l'origine d'environ la moitié de notre exposition d'origine naturelle. La centaine de jours annuelle de patrouille des sousmariniers affectés sur SNLE conduit sans conteste à une diminution de leur exposition naturelle tant externe qu'interne. La quantification précise de cette «non-exposition» demeure irréalisable actuellement. En revanche, même si le prorata temporis n'offre qu'une estimation très grossière, on pourrait évaluer la dose évitée d'origine naturelle à quelques dixièmes de $\mathrm{mSv}$ par an.

Pendant la patrouille concernée par cette étude, les membres de l'équipage ont eu des résultats de dosimétrie passive compris entre $0 \mu \mathrm{Sv}$ et $350 \mu \mathrm{Sv}$. La médiane est à $0 \mu \mathrm{Sv}$.

Pour ces sous-mariniers les doses reçues par exposition professionnelle pendant la patrouille sont donc bien inférieures à celles qui auraient été issues du rayonnement naturel en France s'ils étaient restés à terre (en moyenne de l'ordre de $400 \mu \mathrm{Sv}$ pour deux mois ; Billon, 2004).

\section{Conclusion}

Les anthroporadiamétries comparatives réalisées sur cet échantillon représentatif de l'équipage d'un sous-marin nucléaire lanceur d'engins immédiatement et à 
UNE MOINDRE EXPOSITION DES SOUS-MARINIERS AU RAYONNEMENT NATUREL

distance après le retour de patrouille permettent de démontrer une évidence : l'exposition naturelle par exposition interne est moindre chez les sous-mariniers quand ils sont en patrouille.

\section{RÉFÉRENCES}

Billon S. (2004) Évaluation de l'exposition de la population française à la radioactivité naturelle, Radioprotection 39, 213-232.

Charuau J. (1996) Le radon-222 et ses descendants à vie courte dans l'environnement atmosphérique : origine et méthodes de mesure, Radioprotection 31, 371-388.

Delhomme A. (2001) Sous-mariniers et fécondité ; influence de la vie embarquée à bord des sousmarins nucléaires sur la fécondité des sous-mariniers, Thèse de doctorat en médecine, Lyon.

Laroche P. (2001) Surveillance dosimétrique : résultats des équipages des forces sous-marines de 1989 à 1999, Radioprotection 36, 317-327. 